Pacific Journal of Mathematics

THE FIXED POINT PROPERTY FOR TREELIKE CONTINUA 


\title{
THE FIXED POINT PROPERTY FOR TREE-LIKE CONTINUA WITH FINITELY MANY ARC COMPONENTS
}

\author{
J. B. Fugate AND L. MohleR
}

\begin{abstract}
It is shown that if $M$ is a tree-like continuum with a finite number of arc components, then every continuous mapping of $M$ into itself has a fixed point.
\end{abstract}

A continuum $M$ is a compact, connected metric space. A continuum is said to be tree-like if for every $\varepsilon>0$, there is an $\varepsilon$-cover of $M$ whose nerve is a simple tree (a connected, one-dimensional, acyclic simplicial complex). In [1] Bing raised the question of whether these continua have the fixed point property. This is one of the most famous unsolved fixed point questions for continua. This paper provides an affirmative answer to Bing's question in the case where the tree-like continuum $M$ has finitely many arc components.

Since tree-like continua are hereditarily unicoherent, it is easily seen that any subcontinuum of a tree-like continuum with finitely many arc components has finitely many arc components (see the proof of Lemma 1.3 below). It follows that any such continuum is hereditarily decomposable (indecomposable continua have uncountably many composants). Continua which are hereditarily decomposable and hereditarily unicoherent are called $\lambda$-dendroids. These continua were shown by Cook to be tree-like in [3].

The theorem presented here is thus a special case of the fixed point question for $\lambda$-dendroids which was raised by Knaster in [5]'. Numerous special cases of this question have already been answered. For a survey of these results see [8], Chapter II. In particular Hamilton [4] has shown that all $\lambda$-dendroids have the fixed point property for homeomorphisms and Borsuk [2] has shown that $\lambda$-dendroids which are arcwise connected (dendroids) have the fixed point property for all continuous maps. The theorem presented here generalizes the latter result.

The paper is in two sections. The first section deals with density properties of arc components in $\lambda$-dendroids. Not all of the results in $\S 1$ are required in $\S 2$, which contains the proof of the fixed point theorem. The other material in $\S 1$ is included because the authors feel that it has some independent interest and because it raises a

${ }_{1}$ During revision of this paper for publication in this Journal, the authors received a manuscript from Roman Manka containing a theorem which implies that $\lambda$-dendroids have the fixed point property. 
question which we have been unable to answer.

1. Density properties of arc components of $\lambda$-dendroids.

Definition 1.1. A continuum $M$ is said to be hereditarily decomposable if given any non-degenerate subcontinuum $L$ of $M, L$ can be written as the union of two of its proper subcontinua.

Definition 1.2. A continuum $M$ is said to be hereditarily unicoherent if, given any two subcontinua $P$ and $Q$ of $M, P \cap Q$ is connected.

It is easy to see that if $M$ is a hereditarily decomposable (hereditarily unicoherent) continuum, then every subcontinuum of $M$ is hereditarily decomposable (hereditarily unicoherent). If $p$ and $q$ are two distinct points of a $\lambda$-dendroid $M$, then the fact that $M$ is hereditarily unicoherent implies that there is a unique subcontinuum $l(p, q)$ of $M$ which is irreducible with respect to containing both $p$ and $q$. The uniqueness of $l(p, q)$ implies that if $C$ is any subcontinuum of $M$ which contains both $p$ and $q$, then $l(p, q) \subset C$. The fact that $M$ is hereditarily decomposable implies that $l(p, q)$ is an irreducible continuum of type $\lambda$, i.e., there is a monotone, continuous function $m$ from $l(p, q)$ onto the closed unit interval $[0,1]$ such that $m(p)=0, m(q)=1$ and $m^{-1}(t)$ has void interior in $l(p, q)$ for every $t \in[0,1]$ (see [7], p. 15, Theorem 10). The sets $m^{-1}(t)$ are called tranches of $l(p, q)$. In what follows it is assumed that the reader has some familiarity with the notion of an irreducible continuum of type $\lambda$. For the basic facts concerning these continua the reader is referred to [6], $\S 48$ or [7], Ch. 1 (in [7] irreducible continua of type $\lambda$ are called irreducible continua of type $\left.A^{\prime}\right)$. Of the two accounts, [7] is more compact.

Lemma 1.3. Let $M$ be a $\lambda$-dendroid, $L$ a subcontinuum of $M$ and $A$ an arc component of $M$ which meets $L$. Then $A \cap L$ is an arc component of $L$.

Proof. Let $p \in A \cap L$. If $q$ is in the arc component of $L$ generated by $p$, then clearly $q \in A$. On the other hand, if $q \in A \cap L$, then $l(p, q)$ is an arc since $p, q \in A$. And since $M$ is hereditarily unicoherent, $l(p, q) \subset L$. Thus $q$ is in the arc component of $L$ generated by $p$.

Theorem 1.4. Let $M$ be a $\lambda$-dendroid and let $A_{1}$ and $A_{2}$ be distinct arc components of $M$. Then there is a $G_{\delta}$ subset $G$ of $M$ such that $A_{1} \subset G$ and $A_{2} \cap G=\varnothing$.

Proof. Let $p_{1} \in A_{1}, p_{2} \in A_{2}$ and let $l\left(p_{1}, p_{2}\right)$ be the unique irreducible 
subcontinuum of $M$ from $p_{1}$ to $p_{2}$.

Case 1. $l\left(p_{1}, p_{2}\right)$ has exactly two arc components. Then by 1.3 the two arc components of $l\left(p_{1}, p_{2}\right)$ must be $A_{1}^{\prime}=A_{1} \cap l\left(p_{1}, p_{2}\right)$ and $A_{2}^{2}=A_{2} \cap l\left(p_{1}, p_{2}\right) . \quad l\left(p_{1}, p_{2}\right)$ must have at least one nondegenerate tranche since it is not an arc, and it cannot have more than one since it has only two arc components. Call this tranche $T$. A straightforward argument using the hereditary unicoherence of $M$ will show that if $C$ is any subcontinuum of $M$ which meets both $A_{1}$ and $A_{2}$, then $T \subset C$.

Let $a$ and $b$ be distinct points of $T$ and let $\left\{U_{1}, U_{2}, U_{3}, \cdots\right\}$ and $\left\{V_{1}, V_{2}, V_{3}, \cdots\right\}$ be neighborhood bases for $M$ at $a$ and $b$ respectively such that $U_{n} \cap V_{n}=\varnothing$ for each $n=1,2,3, \cdots$.

Case (1a). $T \subset A_{1}$. In this case, for each natural number $n$ let

$$
C_{n}=\operatorname{cl}\left(\left\{p \in A_{2}: l\left(p, p_{2}\right) \cap U_{n}=\varnothing\right\}\right) .
$$

Then for each $n, C_{n}$, being the closure of an arcwise connected set, is a subcontinuum of $M$. Moreover, since each $C_{n}$ contains $p_{2}$ and does not contain $a$, it must be the case that $C_{n} \cap A_{1}=\varnothing$.

Now let $p \in A_{2}$. Then since $a$ is not an element of the $\operatorname{arc} l\left(p, p_{2}\right)$, there must be an $n$ such that $U_{n} \cap l\left(p, p_{2}\right)=\varnothing$. Thus $p \in C_{n}$. So $\mathrm{U}\left\{C_{n}: n=1,2, \cdots\right\}$ is an $F_{\sigma}$ subset of $M$ which contains $A_{2}$ and fails to meet $A_{1}$. The complement of this set will be the desired set $G$.

Case (1b). $T \subset A_{2}$ (since $T$ has void interior, relative to $l\left(p_{1}, p_{2}\right)$, one can see that this is the only other possible case). In this case for each natural number $n$ let

$$
D_{n}=\operatorname{cl}\left(\left\{p \in A_{2}: l(p, a) \cap V_{n}=\varnothing\right\}\right)
$$

and

$$
E_{n}=\operatorname{cl}\left(\left\{p \in A_{2}: l(p, b) \cap U_{n}=\varnothing\right\}\right) .
$$

By a similar argument to that given for $C_{n}$ in (1a), for each $n, D_{n}$, and $E_{n}$ are subcontinua of $M$ which do not intersect $A_{1}$.

Now let $p \in A_{2}$ and consider the arc $l(p, a)$. If $b \notin l(p, a)$, then there is an $n$ such that $V_{n} \cap l(p, a)=\varnothing$ and we get $p \in D_{n}$. If $b \in$ $l(p, a)$, then $a \notin l(p, b)(l(p, b)$ is a subarc of $l(p, a)$ which does not contain $a)$. So there must be an $n$ such that $U_{n} \cap l(p, b)=\varnothing$ and we get $p \in E_{n}$. The complement of $\bigcup\left\{D_{n} \cup E_{n}: n=1,2, \cdots\right\}$ will thus be the desired set $G$.

Case 2. $l\left(p_{1}, p_{2}\right)$ has more than two arc components. In this 
case let $C$ be an arc component of $l\left(p_{1}, p_{2}\right)$ which contains neither $p_{1}$ nor $p_{2}$ and let $c \in C$. A straightforward unicoherence argument will show that if $F$ is any subcontinuum of $M$ which meets both $A_{1}$ and $A_{2}$, then $c \in F$.

Now let $\left\{W_{1}, W_{2}, W_{3}, \cdots\right\}$ be a neighborhood base for $M$ at $c$ and let

$$
F_{n}=\operatorname{cl}\left(\left\{p \in A_{2}: l\left(p, p_{2}\right) \cap W_{n}=\varnothing\right\}\right) .
$$

An argument similar to that given in (1a) will show that the set $G=M-\bigcup\left\{F_{n}: n=1,2, \cdots\right\}$ has the desired properties. This concludes the proof.

COROLLARY 1.5. If $M$ is a $\lambda$-dendroid with countably many are components, then each arc component of $M$ is a $G_{\dot{j}}$.

Proof. Let $A$ be an arc component of $M$ and number the other arc components of $M ; A_{1}, A_{2}, \ldots$. For each natural number $n$ let $G_{n}$ be a $G_{\delta}$ subset of $M$ which contains $A$ and fails to meet $A_{n}$. Then $A=\bigcap\left\{G_{1}, G_{2}, \cdots\right\}$ is a $G_{\dot{o}}$.

CoRollary 1.6. If $M$ is a $\lambda$-dendroid with finitely many arc components, then each arc component of $M$ is an $F_{o}$.

Proof. Let $A$ be an arc component of $M$. Then $M-A$ is a finite union of $G_{o}$ 's (namely the other arc components of $M$ ) and is thus a $G_{\delta} . A$ is therefore an $F_{\sigma}$.

CoRollary 1.7. If $M$ is a $\lambda$-dendroid with finitely many arc components, then some arc component of $M$ has nonvoid interior.

Proof. This follows immediately from 1.6 and the Baire category theorem.

LEMma 1.8. If $G_{1}$ and $G_{2}$ are disjoint $G_{\tilde{o}}$ subsets of a complete metric space $X$, then $\operatorname{Int}\left(\mathrm{Cl}\left(G_{1}\right)\right) \cap \operatorname{Int}\left(\mathrm{Cl}\left(G_{2}\right)\right)=\varnothing$.

Proof. $V=\operatorname{Int}\left(\mathrm{Cl}\left(G_{1}\right)\right) \cap \operatorname{Int}\left(\mathrm{Cl}\left(G_{2}\right)\right)$ is open in $X$ and thus admits a complete metric (see e.g. [6], p. 408). Moreover, $G_{1} \cap V$ and $G_{2} \cap V$ are dense $G_{o}$ subsets of $V$. Therefore $G_{1} \cap G_{2}$ is dense in $V$ (see e.g. [6], Th. 1, p. 417). But $G_{1} \cap G_{2}=\varnothing$.

CoRollaRy 1.9. If $M$ is a $\lambda$-dendroid with countably many arc components and one arc component $A$ of $M$ is dense, then every other arc component of $M$ is nowhere dense. 
Proof. Let $A^{\prime}$ be another arc component of $M$. Since $A$ is dense, Int $(\mathrm{Cl}(A))=M$. Since $A$ and $A^{\prime}$ are $G_{\delta}$ 's (by 1.5), 1.8 implies that $\operatorname{Int}\left(\mathrm{Cl}\left(A^{\prime}\right)\right)=\varnothing$.

CoRollary 1.10. If $M$ is a $\lambda$-dendroid with countably many arc components, then at most one arc component of $M$ is dense.

Note that if $M$ is a $\lambda$-dendroid with finitely many arc components, then 1.10 follows from 1.7. One might suppose that 1.6 and 1.7 are true of $\lambda$-dendroids with countably many arc components, but in fact this is not the case. The authors have produced an example of a $\lambda$-dendroid with countably many arc components in which every arc component has void interior. The Baire category theorem implies that at least one of the arc components must fail to be an $F_{\sigma}$. The example is produced roughly as follows: Take the cone over the Cantor set and replace the arcs emanating from the accessible points of the Cantor set by $\sin 1 / x$ curves in such a way that (i) the arcs emanating from inaccessible points of the Cantor set are not disturbed. That is, these arcs will have to be "bent" a little so that they will approximate the new $\sin 1 / x$ curves, but they must nevertheless remain arcs. And (ii) the union of the limiting segments of all of the sin $1 / x$ curves is dense in the whole space. A certain amount of delicacy is required in carrying out the above construction. In particular, the diameters of the limiting segments of the sin $1 / x$ curves must tend to 0 .

Thus 1.6 and 1.7 cannot be strengthened. The authors have been unable to answer the question of whether 1.10 can be strengthened to include all $\lambda$-dendroids i.e.,

Question. If $M$ is an arbitrary $\lambda$-dendroid, must $M$ have at most one dense arc component? In view of 1.8 it would suffice to show that distinct arc components of $M$ can be enclosed in disjoint $G_{\delta}$ subsets of $M$. The authors have produced an example of 1-dimensional continuum with exactly two arc components, both of which are dense; but the example is neither hereditarily decomposable nor hereditarily unicoherent (the example is produced by sticking the endpoints of the above example to a "cross-section" of Knaster's indecomposable, chainable continuum with one endpoint).

\section{The fixed point theorem.}

LEMMA 2.1. Let $M$ be a hereditarily unicoherent continuum and let $M_{1}, M_{2}, \cdots, M_{n}$ be subcontinua of $M$. If $M_{i} \cap M_{j} \neq \varnothing$ for all $i, j=$ $1,2, \cdots, n$, then $M_{0}=\bigcap\left\{M_{i}: i=1,2, \cdots, n\right\}$ is a nonvoid subcontinuum of $M$. 
Proof. The proof is by induction on $n$. If $n=2$, the result is obviously true. So let $n \geqq 3$ and suppose that the lemma is true for all integers $k<n$. Let $A=M_{1} \cap M_{2} \cap \cdots \cap M_{n-2}$ and let $B=M_{2} \cap$ $M_{3} \cap \cdots \cap M_{n-1}$. Then by the induction hypothesis and the hereditary unicoherence of $M, A$ and $B$ are nonvoid subcontinua of $M$. Moreover, $A \cap B \neq \varnothing$ (also, by the induction hypothesis). Therefore, $A \cup B$ is a continuum. Thus $(A \cup B) \cap M_{n}$ is a continuum. But $(A \cup B) \cap M_{n}=$ $\left(A \cap M_{n}\right) \cup\left(B \cap M_{n}\right)$ and, once again by the induction hypothesis, $A \cap M_{n} \neq \varnothing \neq B \cap M_{n}$. Therefore, since $\left(A \cap M_{n}\right) \cup\left(B \cap M_{n}\right)$ is connected, we must have $M_{0}=\left(A \cap M_{n}\right) \cap\left(B \cap M_{n}\right) \neq \varnothing$. That $M_{0}$ is a continuum follows directly from the hereditary unicoherence of $M$.

COROLlaRY. Let $M$ be a hereditarily unicoherent continuum, let $\left\{M_{\alpha}: \alpha \in \Gamma\right\}$ be a collection of subcontinua of $M$ and suppose that for all $\alpha, \beta \in \Gamma, M_{\alpha} \cap M_{\beta} \neq \varnothing$. Then $M_{0}=\bigcap\left\{M_{\alpha}: \alpha \in \Gamma\right\}$ is nonvoid subcontinuum of $M$.

Proof. By 2.1 the family $\left\{M_{\alpha}: \alpha \in \Gamma\right\}$ has the finite intersection property. Therefore $M_{0} \neq \varnothing$. Now reindex the family $\left\{M_{\alpha}: \alpha \in \Gamma\right\}$ by some initial sequence of ordinals: $\left\{M_{\alpha}: \alpha<\gamma\right\}$ where $\gamma$ is an ordinal number. For each $\alpha<\gamma$ let $C_{\alpha}=\bigcap\left\{M_{\beta}: \beta<\alpha\right\}$. We want to show that $C_{\gamma}=M_{0}$ is connected. If not, then there is a least ordinal $\alpha_{0}$ such that $C_{\alpha_{0}}$ is not connected. By $2.1 \alpha_{0} \geqq \omega$. Also $\alpha_{0}$ cannot be a successor ordinal. For if $\alpha_{0}=\beta+1$, then $C_{\beta}$ is connected and, since $M$ is hereditarily unicoherent, we get $C_{\alpha_{0}}=C_{\beta} \cap M_{\beta}$ connected. Thus $\alpha_{0}$ is a limit ordinal. But then $C_{\alpha_{0}}=\bigcap\left\{C_{\alpha}: \alpha<\alpha_{0}\right\}$ is the intersection of a nest of continua and is thus a continuum. This contradiction establishes the corollary.

Let $M$ be a $\lambda$-dendroid with finitely many are components and let $f: M \rightarrow M$ be a continuous function. By intersecting a maximal nest of subcontinua $M_{\alpha}$ of $M$ with the property that $f\left(M_{\alpha}\right) \subset M_{\alpha}$ one can find a subcontinuum $M_{0}$ of $M$ such that $f\left(M_{0}\right)=M_{0}$ and no proper subcontinuum of $M_{0}$ is mapped into itself by $f$. Moreover, by Lemma 1.3 $M_{0}$ will have finitely many arc components. The fixed point theorem for $M$ will be established by a series of lemmas. For the remainder of the section $M$ will denote a fixed $\lambda$-dendroid with finitely many arc components and $f$ will denote a continuous mapping of $M$ into itself such that no proper subcontinuum of $M$ is mapped into itself by $f$. By the preceding discussion, it will suffice to show that such $f$ 's have fixed points.

LEMMA 2.2. $f$ maps some arc component of $M$ into itself.

Proof. Since the continuous image of an arcwise connected set 
is arcwise connected, if $A$ is an arc component of $M$, then $f(A)$ will be contained in an arc component of $M$. Moreover, since $f$ is surjective and $M$ has only finitely many arc components, $f$ must permute the arc components of $M$. Let $\mathscr{A}$ be the set of arc components of $M$ and define $f^{*}: \mathscr{A} \rightarrow \mathscr{A}$ by $f^{*}(A)=f(A)$ for each $A \in \mathscr{A}$ (since $f$ is surjective, $f(A)$ must always be an entire arc component). Then $f^{*}$ is a permutation on the finite set $\mathscr{A}$. Now define $\eta$ to be the family of all subsets $N$ of $\mathscr{A}$ satisfying the following conditions:

(1) $\cup N$ is connected,

(2) $\operatorname{cl}(\cup N) \neq M$ and

(3) $N$ is maximal with respect to possessing properties (1) and (2) simultaneously.

We may assume without loss of generality that no arc component of $M$ is dense for if $M$ contains a dense arc component $A$, then 1.10 implies that, since $f(A)$ is dense, $f(A)=A$. Thus $\eta \neq \varnothing$.

Claim 1. If $N \in \eta$, then $f^{*-1}(N) \in \eta$.

Proof of claim. Since $f^{*}$ is a permutation of the finite set $\mathscr{A}$, there must be a natural number $n$ such that $f^{*-1}=f^{* n}$. So $f^{*-1}(N)=$ $f^{* n}(N)$. Therefore, since $\cup N$ is connected, $\cup f^{*-1}(N)=\cup f^{* n}(N)=$ $f^{n}(\cup N)$ is connected. Thus $f^{*-1}(N)$ satisfies condition (1). Since $\operatorname{cl}(\cup N) \neq M$, there is a nonvoid open set $V \subset M-\cup N$. Since $f$ is surjective $f^{-1}(V)$ is a nonvoid open set in the complement of

$$
f^{-1}(\cup N)=\cup f^{*-1}(N) \text {. }
$$

Thus cl $\left(\cup f^{*-1}(N)\right) \neq M$ and $f^{*-1}(N)$ satisfies condition (2).

Finally, suppose that $D \subset \mathscr{A}, f^{*-1}(N) \subsetneq D$, and $\cup D$ is connected. We will complete the proof of the claim by showing that $\operatorname{cl}(\cup D)=M$. Since $f^{*-1}(N) \varsubsetneqq D, N=f^{*}\left(f^{*-1}(N)\right) \varsubsetneqq f^{*}(D)$. Moreover, since $\cup D$ is connected, $\cup f^{*}(D)=f(\cup D)$ is connected. Thus, since $N \in \eta$, $\operatorname{cl}(f(\cup D))=\operatorname{cl}\left(\cup f^{*}(D)\right)=M$. Now choose a natural number $m$ such that $f^{* m}$ is the identity permutation. Then $\operatorname{cl}(\cup D)=\operatorname{cl}\left(\cup f^{* m}(D)\right)=$ $\operatorname{cl}\left(f^{m}(\cup D)\right)=\operatorname{cl}\left(f^{m-1}(f(\cup D))\right)=f^{m-1}(\operatorname{cl}(f(\cup D)))=f^{m-1}(M)=M$. Thus $f^{*-1}(N)$ satisfies condition (3).

Claim 2. If $N_{1}, N_{2} \in \eta$, then $\operatorname{cl}\left(\cup N_{1}\right) \cap \operatorname{cl}\left(\cup N_{2}\right) \neq \varnothing$.

Proof of claim. Just suppose that $N_{1}, N_{2} \in \eta$ and

$$
\operatorname{cl}\left(\cup N_{1}\right) \cap \operatorname{cl}\left(\cup N_{2}\right)=\varnothing \text {. }
$$

Let $F \in \mathscr{A}$ such that $F \notin N_{1}$ and $\cup N_{1} \cup F$ is connected (such a set must exist, since $M$ is connected). Now since $N_{1} \in \eta, \operatorname{cl}\left(\cup N_{1} \cup F\right)=$ 
M. Thus $\operatorname{cl}\left(\cup N_{2}\right) \subset \operatorname{cl} F$ and so $\left(\cup N_{2}\right) \cap F$ is connected. Since $N_{2} \in \eta$, $\operatorname{cl}\left(\cup N_{2} \cup F\right)=M$. But $\operatorname{cl}\left(\cup N_{2}\right) \subset \operatorname{cl} F$, so $M=\operatorname{cl}\left(\cup N_{2}\right) \cup \operatorname{cl}(F)=\operatorname{cl} F$, violating the assumption that no arc component of $M$ is dense. This establishes the second claim.

Now Lemma 2.1 and Claim 2 imply that $M_{0}=\cap\{\operatorname{cl}(\cup N): N \in \eta\}$ is a subcontinuum of $M$. And condition (2) on $\eta$ implies that $M_{0}$ is a proper subcontinuum. Moreover, if $N$ is any element of $\eta$, then Claim 1 implies that there is an $N_{1} \in \eta$ (namely $f^{*-1}(N)$ ) such that

$$
f\left(\cup N_{1}\right) \subset \cup N .
$$

This in turn implies that $\cap\{\operatorname{cl}(f(\cup N)): N \in \eta\} \subset \cap\{\operatorname{cl}(\cup N): N \in \eta\}$. Thus we get

$$
\begin{aligned}
f\left(M_{0}\right) & =f(\cap\{\operatorname{cl}(\cup N): N \in \eta\}) \subset \cap\{f(\operatorname{cl}(\cup N)): N \in \eta\} \\
& =\cap\{\operatorname{cl}(f(\cup N)): N \in \eta\} \subset \cap\{\operatorname{cl}(\cup N): N \in \eta\}=M_{0},
\end{aligned}
$$

contradicting one of the original assumptions about $f$. This concludes the proof.

THEOREM 2.3. If $M$ is a $\lambda$-dendroid and the collection of arc components of $M$ is finite, then $M$ has the fixed point property.

Proof. Suppose, to the contrary, that there is a map $f: M \rightarrow M$ such that $f$ has no fixed point. As explained at the beginning of this section, we may assume that $f$ maps $M$ onto $M$ and that no proper subcontinuum of $M$ is mapped into itself. From Lemma 2.2, we conclude that there is an arc component $A$ of $M$ such that $f[A]=$ $A$. Then $f[\operatorname{cl} A]=\operatorname{cl} f[A]=\operatorname{cl} A$, so $\operatorname{cl} A$ is a subcontinuum of $M$ which is mapped into itself. Thus cl $A=M$.

Choose $p \in A$. Since $p \neq f(p)$ and $f(p) \in A$, the continuum irreducible from $p$ to $f(p), l(p, f(p))$ is an arc. Using the uniform continuity of $f$, we see that there is a point $b \in l(p, f(p)), p \neq b$, such that the arc $l(p, b)$ misses its image under $f$.

Claim. If $x \in l(p, b)$, then $x \in l(p, f(x))$. Suppose that there is a point $x \in l(p, b)$ such that $x \notin l(p, f(x))$. Since $f[l(p, b)]$ is arcwise connected, there is an arc $J$, from $f(x)$ to $f(p)$, with $J \subset f[l(p, b)]$. Clearly, $J \cap l(p, b)=\varnothing$, and $x \notin J$. Thus $J \cup l(p, f(x))$ contains an are $H$ from $p$ to $f(p)$. Since $x \notin J \cup l(p, f(x)), x \notin H$. Hence, there arc two arcs from $p$ to $f(p)$, namely one containing $x$ and $H$. This contradicts the hereditary unicoherence of $M$ and establishes the claim.

Let $\mathscr{L}=\{l(p, q): q \in A$, and if $x \in l(p, q)$, then $x \in l(p, f(x))\}$. Now $\mathscr{L}$ is partially ordered by inclusion and $\{l(p, b)\}$ is a nest in $\mathscr{L}$. By the maximal principle, there is a maximal nest $\mathscr{N}$ in $\mathscr{L}$ which 
contains $\{l(p, b)\}$. Suppose that $\Lambda$ is an index set such that $\mathscr{N}=$ $\left\{l\left(p, q_{\lambda}\right): \lambda \in \Lambda\right\}$. Let $L=\cup\left\{l\left(p, q_{\lambda}\right): \lambda \in \Lambda\right\}$. Then $L$ is arcwise connected and $\mathrm{cl} L$ is a continuum. Note that if $x \in L$, then $x \in l(p, f(x))$.

Moreover, $\mathrm{cl} L$ is irreducible from $p$ to some point. For if this is not true, then $\operatorname{cl} L$ is the union of two of its proper subcontinua $D$ and $E$, such that $p \in D \cap E$. Since $L$ is dense in cl $L$, there are elements $\lambda, \mu \in \Lambda$ such that $l\left(p, q_{\lambda}\right) \cap(D-E) \neq \varnothing$ and $l\left(p, q_{\mu}\right) \cap(E-$ $D) \neq \varnothing$. Inasmuch as $\mathscr{N}$ is a nest, are we may assume that $l\left(p, q_{\lambda}\right) \subset$ $l\left(p, q_{\mu}\right)$. Since $M$ is hereditarily unicoherent, $l\left(p, q_{\mu}\right) \cap D$ and $l\left(p, q_{\mu}\right) \cap$ $E$ are continua. Thus, $l\left(p, q_{\mu}\right)$ is the union of two of its proper subcontinua, both of which contain $p$. Thus $p$ is not a point of irreducibility of $l\left(p, q_{\mu}\right)$. This is impossible, so $\mathrm{cl} L$ is irreducible from $p$ to some point.

Let $K=\{y: y \in M$ and cl $L$ is irreducible from $p$ to $y\}$. Since $M$ is hereditarily decomposable, it follows from [7, Th. 7, p. 13] that $K$ is a continuum. Furthermore, $K \cap A=\varnothing$. For otherwise, there is a point $t \in K \cap A$, and so $l(p, t)$ is an arc from $p$ to $t$. This means that $K=\{t\}$ and so $\operatorname{cl} L=l(p, t)$. Thus $\mathrm{cl} L=L \cup\{t\}$. It is not difficult to see that $t \in l(p, f(t))$, since there are points of $L$ arbitrarily close to $t$. This means that $l(p, t)$ is a largest element of the nest $\mathscr{N}$. However, the same sort of argument as was used to establish the existence of the arc $l(p, b)$, to start the construction of $\mathscr{N}$, will show that $\mathscr{N}$ cannot have a largest element. Thus $\operatorname{cl} L=L \cup K$ and $A \cap \operatorname{cl} L=L$.

\section{Claim. $f[K] \cap K \neq \varnothing$.}

If this fails, then there are disjoint open sets $U$ and $V$ containing $K$ and $f[K]$ respectively. We may assume that $U$ was chosen small enough that $f[U] \subset V$. Now $\mathrm{cl} L-U$ is closed and a subset of $A$. Thus $f[\operatorname{cl} L-U] \subset f[A]=A$, so $f[\operatorname{cl} L-U] \cap K=\varnothing$. So there is an open set $W$ with $K \subset W \subset U$ and $W \cap f[\mathrm{cl} L-U]=\varnothing$. Since $f[U] \subset V$ and $V \cap W=\varnothing$, it follows that $f[\mathrm{cl} L] \cap W=\varnothing$.

Since $l(p, f(p))$ is an arc in $L$, we see that $W-l(p, f(p))$ is an open set containing $K$. Each point of $K$ is a limit point of $L$, so we may choose $x \in(W-l(p, f(p))) \cap L$. Clearly, $f[l(p, x)]$ is an arcwise connected continuum containing $f(p)$ and $f(x)$. Thus $l(p, f(p)) \cup f[l(p, x)]$ is an arcwise connected continuum containing $p$ and $f(x)$, hence containing the unique arc from $p$ to $f(x), l(p, f(x))$. Because $x \in L$, we must have $x \in l(p, f(x))$, so $x \in l(p, f(p)) \cup f[l(p, x)]$. However, $x \notin$ $l(p, f(p))$, by choice of $x$, so $x \in f[l(p, x)]$.

Let $z \in l(p, x)$ with $f(z)=x$. Now $l(p, x) \subset L$, so $z \in L$. Thus $x=f(z) \in f[\mathrm{cl} L] \cap W$, which contradicts the concluding statement of the first paragraph and establishes the claim. 
Since $f$ permutes the arc components of $M$, it follows that $f[M-$ $A]=M-A$. Since $K \subset M-A$, there is a component $D$ of $M-A$ such that $K \subset D$. Now $f[D]$ is contained in a component of $M-A$; because $K \cap f[K] \neq \varnothing$, we have $f[D] \cap D \neq \varnothing$, so this component of $M-A$ can only be $D$ itself; that is $f[D] \subset D$. Thus $f[\operatorname{cl} D]=\operatorname{cl} f[D]=\subset \operatorname{cl} D$, so $\operatorname{cl} D$ is a subcontinuum of $M$ which is mapped into itself. Since $A$ is a dense arc-component of $M$, Corollary 1.7 guarantees that Int $A \neq \varnothing$. Now $D \subset M-A$, so cl $D \neq M$. Thus cl $D$ is a proper subcontinuum of $M$ which is mapped into itself. This contradiction concludes the proof.

\section{REFERENCES}

1. R. H. Bing, Snake-like continua, Duke Math. J., 18 (1951), 653-663.

2. K. Borsuk, $A$ theorem on fixed points, Bull. Acad. Polon. Sci., 2 (1954), 17-20.

3. H. Cook, Tree likeness of dendroids and $\lambda$-dendroids, Fund. Math., 68 (1970), 19-22.

4. 0. H. Hamilton, Fixed points under transformations of continua which not are connected im kleinen, Trans. Amer. Math. Soc., 44 (1938), 18-24.

5. B. Knaster, Problem 526 (dated Nov. 22, 1960), New Scottish Problem Book.

6. K. Kuratowski, Topology, vols. I, II, Academic Press, New York, 1966, 1968.

7. E. S. Thomas, Jr., Monotone Decompositions of Irreducible Continua, Rozprawy Matematyczny L., 1966.

8. T. Van der Walt, Fixed and Almost Fixed Points, Mathematical Centre Tracts, Volume 1, Mathematisch Centrum, Amsterdam, 1963.

Received August 1, 1974 and in revised form November 11, 1974.

UNIVERSITY OF KENTUCKY

AND

Suny at Buffalo 


\section{PACIFIC JOURNAL OF MATHEMATICS}

\section{EDITORS}

RICHARD ARENS (Managing Editor)

University of California

Los Angeles, California 90024

\section{J. DugundJI}

Department of Mathematics University of Southern California Los Angeles, California 90007

D. Gilbarg and J. Milgram

Stanford University

Stanford, California 94305
University of Washington Seattle, Washington 98105

\section{ASSOCIATE EDITORS}
E. F. BECKENBACH
B. H. NeumanN
F. WolF
K. YoShIDA

\section{SUPPORTING INSTITUTIONS}

\author{
UNIVERSITY OF SOUTHERN CALIFORNIA \\ STANFORD UNIVERSITY \\ UNIVERSITY OF TOKYO \\ UNIVERSITY OF UTAH \\ WASHINGTON STATE UNIVERSITY \\ UNIVERSITY OF WASHINGTON \\ $\stackrel{*}{*} \stackrel{*}{*} \stackrel{*}{ }{ }^{*}$ AMERICAN MATHEMATICAL SOCIETY
}

The Supporting Institutions listed above contribute to the cost of publication of this Journal, but they are not owners or publishers and have no responsibility for its content or policies.

Mathematical papers intended for publication in the Pacific Journal of Mathematics should be in typed form or offset-reproduced, (not dittoed), double spaced with large margins. Underline Greek letters in red, German in green, and script in blue. The first paragraph or two must be capable of being used separately as a synopsis of the entire paper. Items of the bibliography should not be cited there unless absolutely necessary, in which case they must be identified by author and Journal, rather than by item number. Manuscripts, in triplicate, may be sent to any one of the editors. Please classify according to the scheme of Math. Reviews, Index to Vol. 39. All other communications should be addressed to the managing editor, or Elaine Barth, University of California, Los Angeles, California, 90024.

The Pacific Journal of Mathematics expects the author's institution to pay page charges, and reserves the right to delay publication for nonpayment of charges in case of financial emergency.

100 reprints are provided free for each article, only if page charges have been substantially paid. Additional copies may be obtained at cost in multiples of 50 .

The Pacific Journal of Mathematics is issued monthly as of January 1966. Regular subscription rate: $\$ 72.00$ a year (6 Vols., 12 issues). Special rate: $\$ 36.00$ a year to individual members of supporting institutions.

Subscriptions, orders for back numbers, and changes of address should be sent to Pacific Journal of Mathematics, 103 Highland Boulevard, Berkeley, California, 94708.

\section{PUBLISHED BY PACIFIC JOURNAL OF MATHEMATICS, A NON-PROFIT CORPORATION}

Printed at Kokusai Bunken Insatsusha (International Academic Printing Co., Ltd.), 270, 3-chome Totsuka-cho, Shinjuku-ku, Tokyo 160, Japan.

\section{Copyright (C) 1975 by Pacific Journal of Mathematics} Manufactured and first issued in Japan 


\section{Pacific Journal of Mathematics}

\section{Vol. 57, No. $2 \quad$ February, 1975}

Norman Larrabee Alling, On Cauchy's theorem for real algebraic curves with boundary .......

Daniel D. Anderson, A remark on the lattice of ideals of a Prüfer domain ..................

Dennis Neal Barr and Peter D. Miletta, A necessary and sufficient condition for uniqueness of

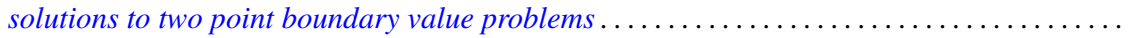

Ladislav Beran, On solvability of generalized orthomodular lattices . . . . . . . . . . ........

L. Carlitz, A three-term relation for some sums related to Dedekind sums . . . . . . . . . .....

Arthur Herbert Copeland, Jr. and Albert Oscar Shar, Images and pre-images of localization

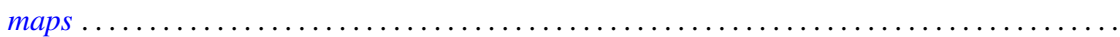

G. G. Dandapat, John L. Hunsucker and Carl Pomerance, Some new results on odd perfect

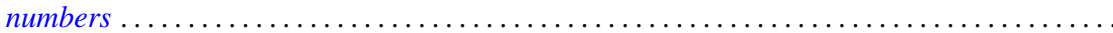

M. Edelstein and L. Keener, Characterizations of infinite-dimensional and nonreflexive

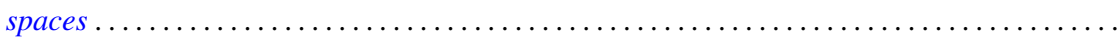

Francis James Flanigan, On Levi factors of derivation algebras and the radical embedding

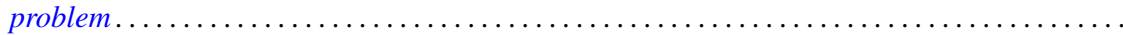

Harvey Friedman, Provable equality in primitive recursive arithmetic with and without

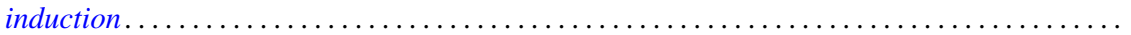

Joseph Braucher Fugate and Lee K. Mohler, The fixed point property for tree-like continua with

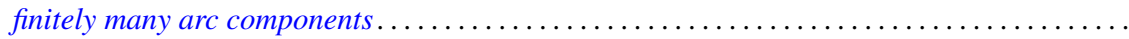

John Norman Ginsburg and Victor Harold Saks, Some applications of ultrafilters in

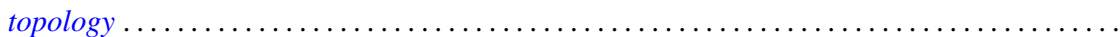

Arjun K. Gupta, Generalisation of a "square" functional equation .....................

Thomas Lee Hayden and Frank Jones Massey, Nonlinear holomorphic semigroups ..........

V. Kannan and Thekkedath Thrivikraman, Lattices of Hausdorff compactifications of a locally

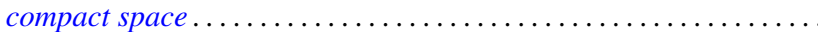

J. E. Kerlin and Wilfred Dennis Pepe, Norm decreasing homomorphisms between group

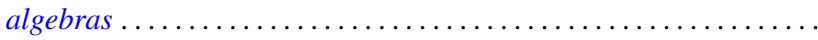

Young K. Kwon, Behavior of $\Phi$-bounded harmonic functions at the Wiener boundary ...

Richard Arthur Levaro, Projective quasi-coherent sheaves of modules .

Chung Lin, Rearranging Fourier transforms on groups...........................

David Lowell Lovelady, An asymptotic analysis of an odd order linear differential equation . . 4475

Jerry Malzan, On groups with a single involution .......................... 481

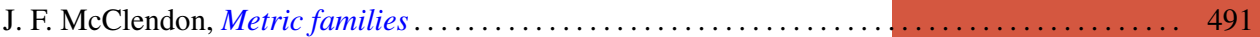

Carl Pomerance, On multiply perfect numbers with a special property .

Mohan S. Putcha and Adil Mohamed Yaqub, Polynomial constraints for finiteness of semisimple rings. .

Calvin R. Putnam, Hyponormal contractions and strong power convergence . . . . . . . . . 531

Douglas Conner Ravenel, Multiplicative operations in $\mathrm{BP} * \mathrm{BP} \ldots \ldots \ldots \ldots \ldots \ldots \ldots \ldots \ldots .539$

Judith Roitman, Attaining the spread at cardinals which are not strong limits . . . . . . . . . 545

Kazuyuki Saitô, Groups of *-automorphisms and invariant maps of von Neumann algebras . . . 553

Brian Kirkwood Schmidt, Homotopy invariance of contravariant functors acting on smooth

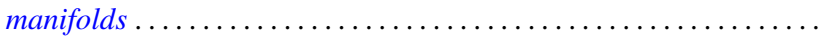

Kenneth Barry Stolarsky, The sum of the distances to $N$ points on a sphere.

Mark Lawrence Teply, Semiprime rings with the singular splitting property.

J. Pelham Thomas, Maximal connected Hausdorff spaces..............

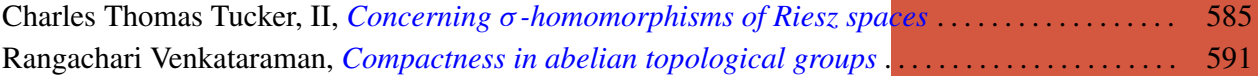

William Charles Waterhouse, Basically bounded functors and flat sheaves . . . . . . . . . . . 597

David Westreich, Bifurcation of operator equations with unbounded linearized part ......... 611

William Robin Zame, Extendibility, boundedness and sequential convergence in spaces of 